Research Article

\title{
Needleless Electrospinning and Electrospraying of Mixture of Polymer and Aerogel Particles on Textile
}

\author{
Abu Shaid $(\mathbb{D}$, Lijing Wang $(\mathbb{D}$, Rajiv Padhye $(\mathbb{D}$, and Amit Jadhav \\ School of Fashion and Textiles, RMIT University, Melbourne, VIC, Australia \\ Correspondence should be addressed to Abu Shaid; s3304096@student.rmit.edu.au \\ Received 7 January 2018; Revised 13 April 2018; Accepted 17 April 2018; Published 23 May 2018 \\ Academic Editor: Frederic Dumur \\ Copyright (c) 2018 Abu Shaid et al. This is an open access article distributed under the Creative Commons Attribution License, \\ which permits unrestricted use, distribution, and reproduction in any medium, provided the original work is properly cited.

\begin{abstract}
Needleless electrospinning and electrospraying of aerogel particles in comparatively lower electric voltage $(9 \mathrm{kV})$ have been demonstrated in the paper. Aerogel particles were dispersed in polymer solution and then needlelessly electrospun/sprayed by creating high electric charge at the syringe tip using a curved wire. FTIR spectra and SEM images proved that aerogel particles were deposited onto the base textile. In case of electrospinning, a nanofibre web holding the aerogel particles covered the fabric surface, whereas in case of electrospraying, aerogel particles deposited with the microdroplets of the polymer. The electrospraying process showed great potential for fabric surface functionalization due to the high amount of particle deposition on fabric. The new approach can be applicable for transferring other particulate materials on fabric surface through needleless electrospinning
\end{abstract} \\ and electrospraying processes.
}

\section{Introduction}

Electrospinning is a method where high electric force is used as a mean to pull polymer solution and to produce very fine fibres with the diameter in nanometre range. On the contrary, if the polymer molecules do not have sufficient molecular cohesion to withstand the electric pull to form nanofibre, then the ejected stream breaks and creates drops of polymer and the process is referred as electrospraying. Though the method of electrospinning was invented in 1934 [1], it took more than 40 years to come to the needleless method of electrospinning. The first needleless electrospinning was based on a ring spinneret [2], and gradually various other shapes of spinneret were evolved. In the most basic electrospinning setup, a high voltage is applied to a metallic needle and polymer solution is extruded through the hole of the needle. The voltage is continuously increased to the point when the repulsive force between same charge molecules exceeds the surface tension and a jet of polymer solution erupts towards the grounded collector. The ejected polymer jets experience instability in their path to the collector which causes bending and stretching of the jet and results in the production of very fine fibres. In needleless electrospinning method, the needle is replaced with roller, ball, disc, cylinder, beaded wire, and so on. All these needleless electrospinning methods can be divided primarily into two groups: rotary and stationary spinneret needleless electrospinning [3]. Rotary needleless electrospinning includes rotary cylinder, disc, ball, spiral wire coil, splashing spinneret, cone, edge of metal plate, rotating roller, bowel edge, rotary beaded chain [4-9], and so on. In stationary spinneret needleless electrospinning, the use of vertical rod [10], air bubble [11], conical wire coil [12], and metallic tube electrode [13] has been reported. In current approach, we used a curved wire inside the syringe and syringe tip to condense high electric charge inside the tip where the mixture of polymer and aerogel particles gets charged and extruded to the collector.

Aerogel is a nanoporous material with extremely light weight, high heat insulation, and impressive absorption capability which can be utilized in numerous fields of application like heat protection, oil absorption, and acoustic insulation. Application of aerogel on textiles is a growing interest, and gradually the field of application is expanding. There are few possible ways how the aerogel can be combined with textiles. Textile fibre, yarn, or fabric can be added 
into the aerogel precursor solution during its synthesis, or already synthesized aerogel can be applied on fibre, yarn, or fabric surface. There are plenty of literatures available where the fibres are immersed into the silica solution to prepare fibrous aerogel composite $[14,15]$. In case of the use of synthesized aerogel on textiles, the aerogel particles are mostly used to maintain the flexibility of textile materials. Several reports are available where aerogel particles are either mixed with binder and knife coated on textiles $[16,17]$, or padded with textile material [18], or thermally bonded in nonwoven textile [19].

A very limited number of reports are available where the electrospinning technique was used to combine both aerogel and textile $[20,21]$. Such approach is mainly to add electrospun nanofibre into the aerogel precursor before aerogel synthesis to produce fibrous aerogel. To the best of our understanding, there was no literature available regarding the use of synthesized aerogel particles in polymer solution to electrospin aerogel containing nanofibre until 2015. The application of aerogel particles in polymer solution to produce nanofibre has recently been reported by MazroueiSebdani et al. [22]. Here, aerogel particles were mixed with polymer solution and extruded through the hole of the electrospinning needle by using high voltage. For the first time, the current study aims to apply aerogel particle on textile surface by using an innovative needleless electrospinning and electrospraying method. Without limiting the polymer type, here one water-based polymer and one solvent-based polymer solution were used to demonstrate the concept of such electrospinning technique. In traditional needled electrospinning of particles, clogging of the needle hole is a major concern, whereas the needleless method requires very high voltage as high as $120 \mathrm{kV}$ [23]. In this respect, the current method has a significant advantage that it is a needleless approach and requires comparatively lower power supply $(9 \mathrm{kV})$.

\section{Experimental}

2.1. Chemicals. Laboratory-grade dimethylformamide (DMF) was purchased from Merck KGaA (Germany), PVA (polyvinyl alcohol) was obtained from Chem-Supply (Australia), thermoplastic polyurethane (TPU) chip was purchased from $\mathrm{Pa}-$ cific Urethanes (Australia), and Enova aerogel particles were sourced from Cabot Corporation (USA). 100\% Nomex woven fabric was used as base textile. Cationic surfactant Parudul BS was used to disperse aerogel particles. Aerogel particles were grinded to smaller size by using the ball milling method prior to the addition in the polymer solution. 9\% PVA polymer solution was prepared in deionised water, and then, the small amount of binder material (Tubicoat WP1665 HT) was added in the solution. 5\% TPU solution was prepared in DMF at $40^{\circ} \mathrm{C}$ and continued to be stirred for 8 hours.

2.2. Instruments. A KDS 200 digital syringe pump (from KD Scientific Inc., Holliston, MA, USA) was used to maintain a constant flow of solution. High voltage power supply, ES30P-5W, with a maximum output voltage of $30 \mathrm{kV}$ was used to generate high voltage. A $10 \mathrm{ml}$ plastic syringe was used. A solid metallic wire of $0.8 \mathrm{~mm}$ diameter was inserted into the syringe or the tip of the syringe in a formation of loop while the tail of the loop was extended into the solution. The morphology of the fabric after electrospinning and spraying was observed using a FEI Quanta 200 scanning electron microscope (SEM). A PerkinElmer 400 FTIR spectrometer was used for the characterisation of the sample within the range of $650 \mathrm{~cm}^{-1}$ and $4000 \mathrm{~cm}^{-1}$.

2.3. Needleless Electrospinning Method. A mixture of $1.5 \%$ aerogel and polymer solution was prepared by very slow addition of aerogel particles in the polymer solution with continuous stirring. Then, few drops of ionic surfactant and binder were added, and the mixture was continued to be stirred for at least 4 hours before electrospinning. As shown in Figure 1, a metallic wire was inserted into the tip of a plastic syringe where the tail of the wire was extended to the polymer mix and a curved loop was formed inside the tip. The wire was connected with the high voltage source. The highly charged wire inside the polymer mix charged the molecules before reaching to the tip while the curved wire loop in the tip condensed high electric charge. A rotating drum collector, covered with Nomex fabric as base textile, was attached with a grounded wire. The collector was placed at $8 \mathrm{~cm}$ working distance from the syringe tip. Aerogel and polymer mixture was supplied through the plastic syringe, and the syringe piston was attached with the movable jaw of the metering pump to maintain a constant rate of liquor flow. When the high voltage source was turned on, high electric charges were condensed on the curved wire inside the syringe tip and charged the polymer-aerogel mixture. As soon as the repulsive force of similar charges exceeded the surface tension (at $9 \mathrm{kV}$ ), thin polymer jets with dispersed aerogel particles were ejected towards the collector as shown in Figure 2. The whipping instability thinned the polymer jet and produced nanofibres. As the aerogel particles were also ejected with the polymer jet, the produced nanofibre webs deposited on the particles and bonded them on base textile.

2.4. Needleless Electrospraying. The same apparatus was used to electrospray aerogel particle on fabric surface. To electrospray rather than electrospinning, a lower concentration of polymer solution was used. In this case, $1 \%$ aerogel particle was dispersed in 5\% TPU solution in DMF. The aerogel-polymer mixture jet is ejected by the high electric pull but breaks into numerous droplets while travelling towards the collector due to the lack of sufficient molecular cohesion. Aerogel-mixed polymer droplets deposited on the base fabric surface and fixed therein by the physical bonding of polymer and fabric.

2.5. Measurement of Fibre Diameter and Particle Size. The particle size of transferred aerogel particle and the diameter of formed nanofibre were measured by using Image ${ }^{\circledR}$, a recognized software for porosity, particle sizing, and measuring in an image. 


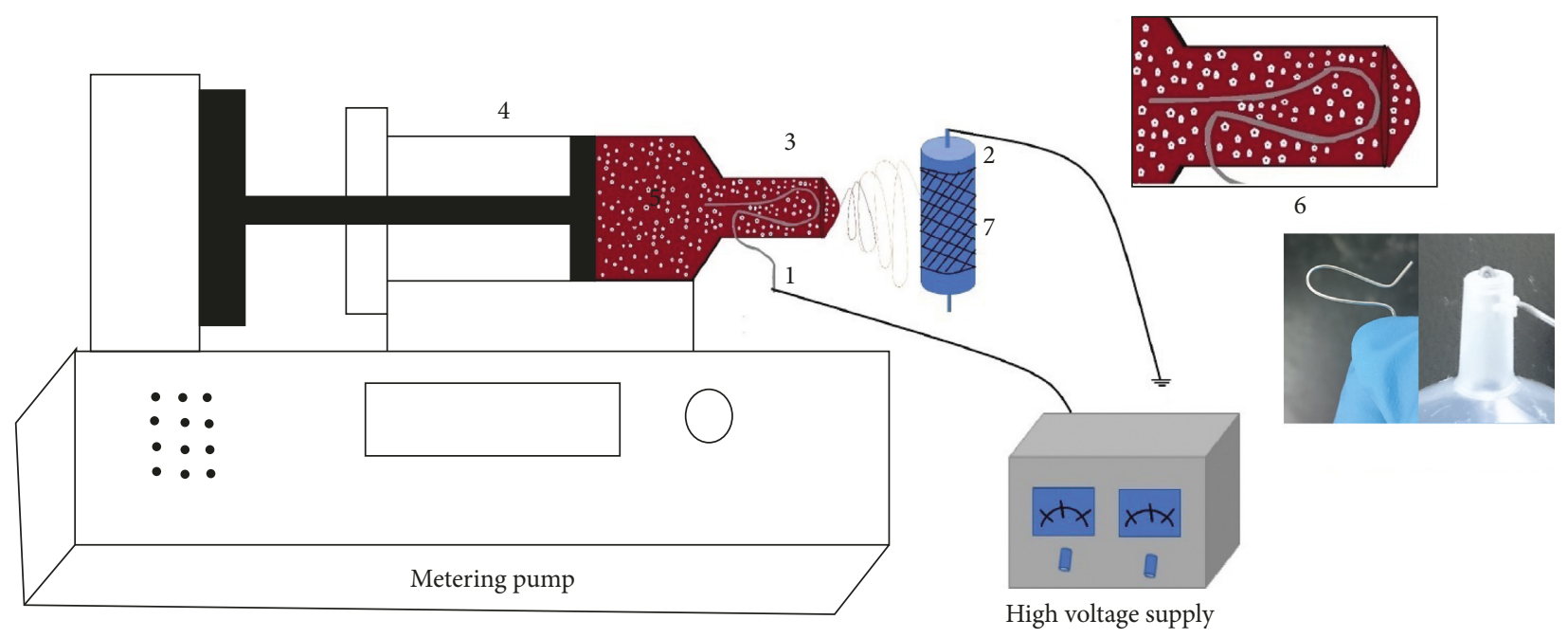

Figure 1: Schematic diagram of the process setup. A curved wire (1) was inserted inside the syringe tip (3). The high voltage source was connected to this wire. The textile fabric (7) was wrapped around the rotating drum collector (2) to act as base textile. The syringe (4) was placed on the metering pump. When the high voltage was turned on, aerogel containing polymer mix (5) became charged and attracted towards the collector. The syringe tip with wire is shown insight (6).

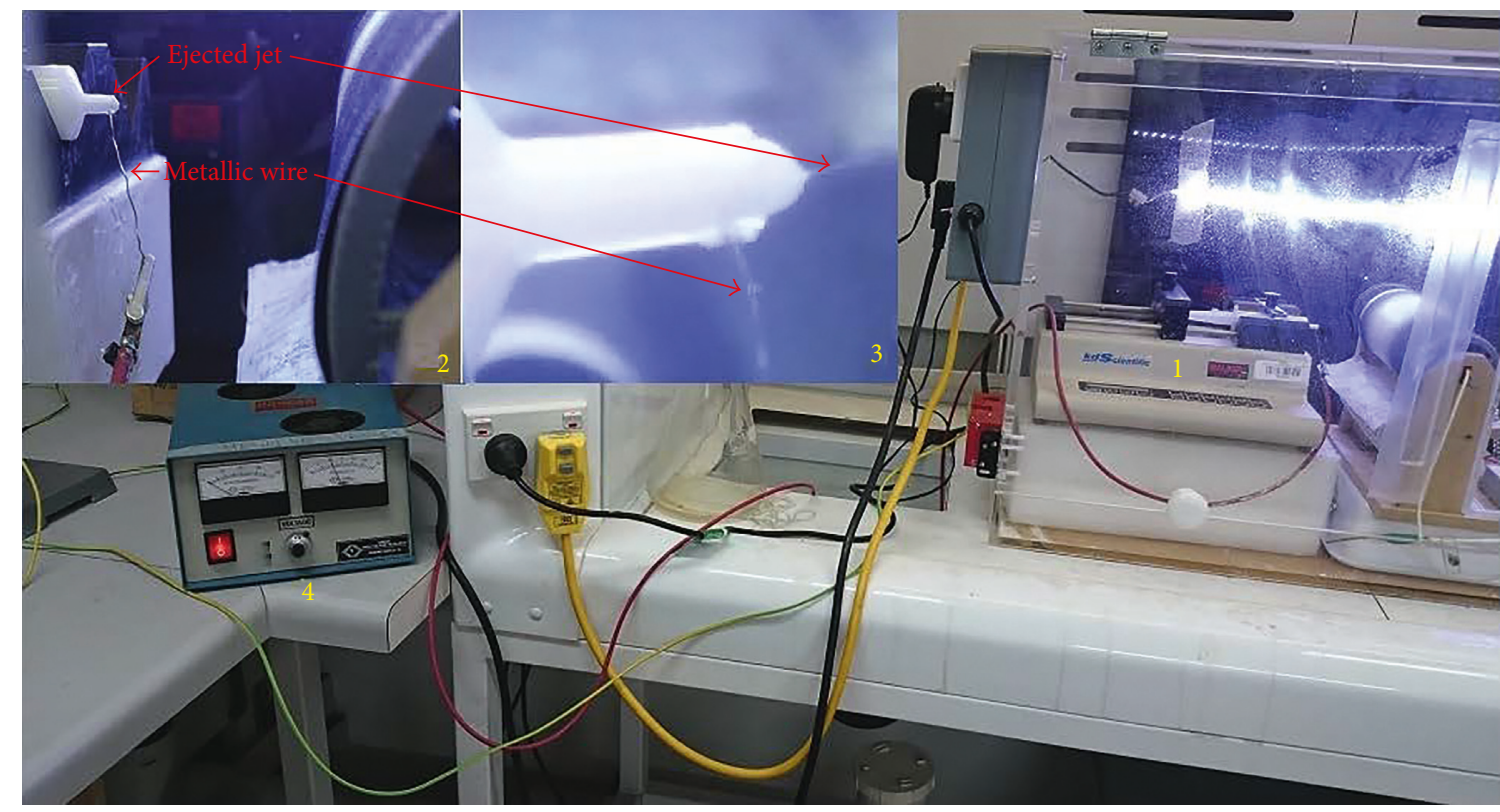

FIGURE 2: Electrospinning/spraying setup. The plastic syringe was placed attached with the arm of the metering pump (1) and connected to the high voltage source (4). A rotating drum collector (2) was connected to the grounded wire. When the voltage was turned on, the polymer jet was ejected (3) from the syringe tip.

\section{Results and Discussion}

3.1. Transfer of Aerogel Particles. The fabric sample after electrospinning showed a white layer of nonwoven web on base textile. When this sample was observed under the SEM, electrospun nanofibres were clearly visible with randomly deposited aerogel particles.

It was observed that aerogel particles have been transferred through electrical force along with nanofibres, deposited onto the base textile, and bonded therein with the nanofibre webs as hundreds of nanofibres covered the particles to hold them on the fabric surface (Figure 3 ). Thus, the aerogel particles were trapped between woven base fabric and nonwoven electrospun nanofibre web. Like any other usual electrospun web, here aerogel nonwoven was formed as layers of electrospun web on base fabric. In general, the fastness of such type of deposition will depend on the features of the polymer (chemical composition, molecular weight, solid content, etc.), the surface characteristics of the base textile (fibre type, fabric construction, surface finish, etc.), and electrospinning parameters. Thus, the bonding strength will totally depend on the selection of material and process parameters chosen by any individual. Moreover, in many applications (such as filtration), the 


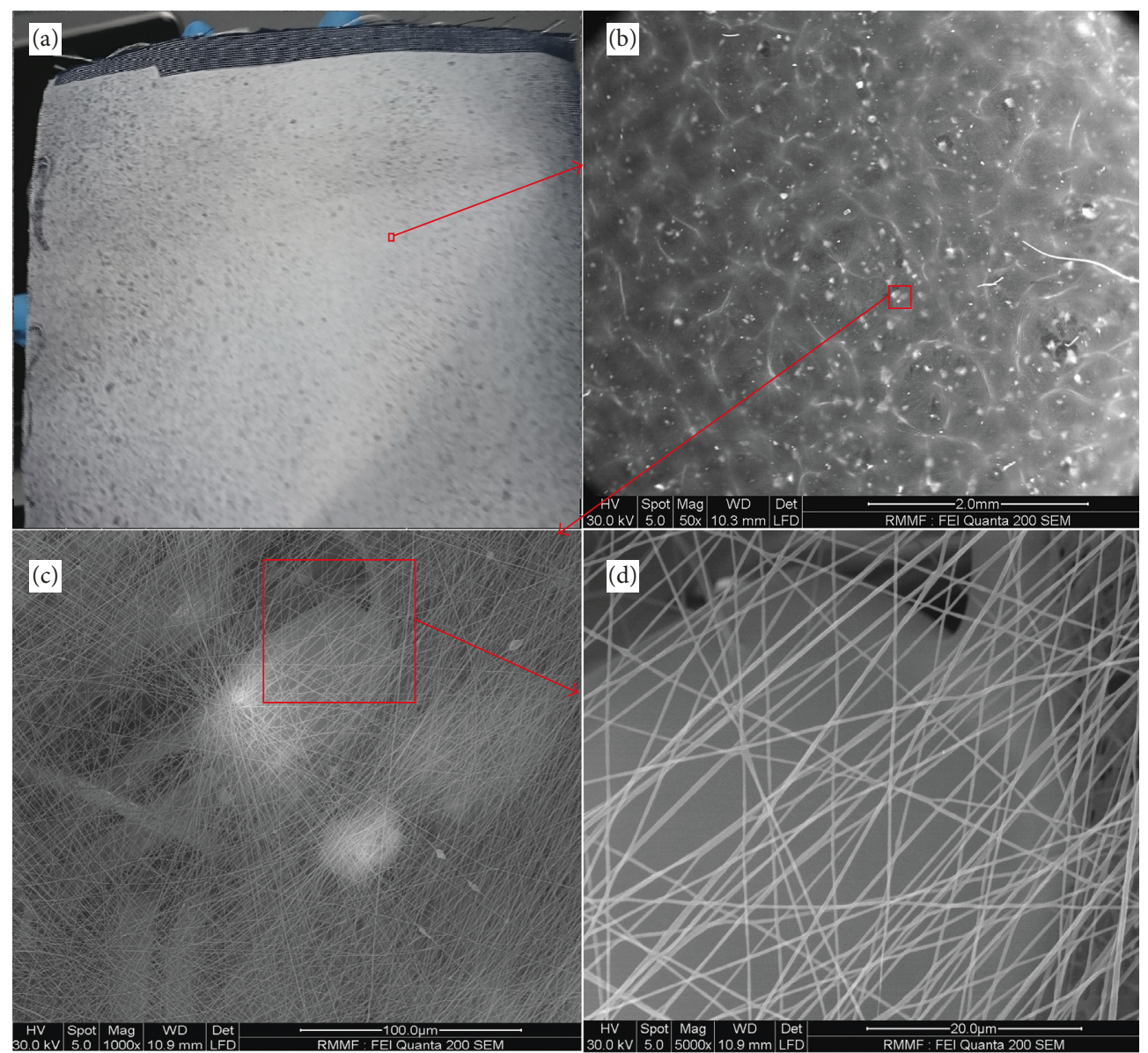

Figure 3: Electrospun layer on base textile in normal view (a), under SEM (b), and at higher magnifications (c, d).

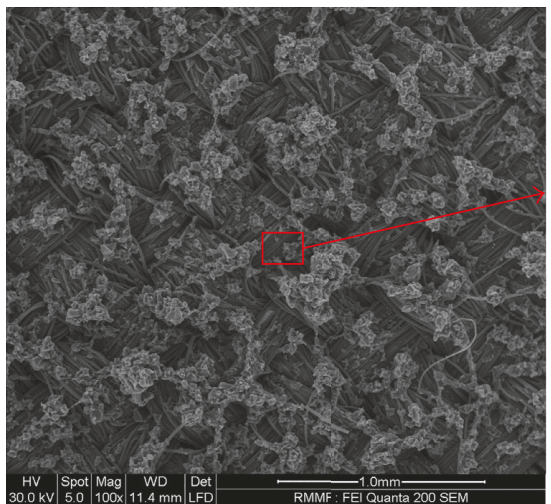

(a)

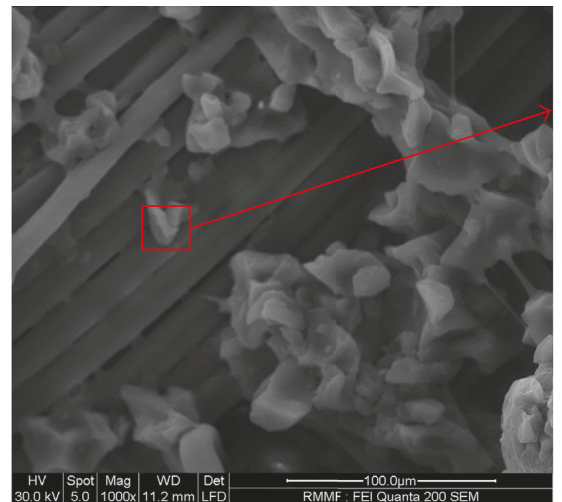

(b)

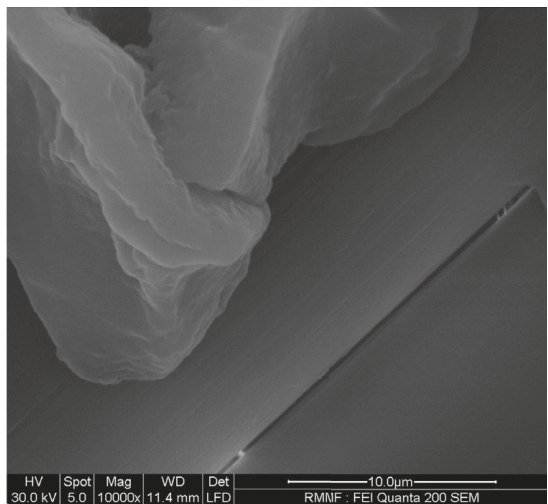

(c)

FIGURE 4: Electrosprayed aerogel particles on fabric surface. Higher magnification shows the sprayed drops of polymer containing numbers of aerogel particles.

firm bonding of deposited nonwoven layers is not a major concern as the nonwoven stays between other layers of fabric or material in sandwich construction. In any case, it is obvious from the above discussion that the developed method is an efficient way to needlelessly electrospin aerogel particles and with comparatively lower voltage.
In case of electrospraying, aerogel particles were transferred through the microdrops of the polymer. In Figure 4, the SEM micrograph clearly shows numerous such drops on fabric surface covering almost entire base textile. To ensure that the transferred drops are not just the polymer clots, the FTIR analysis had been carried out. Figure 5 shows the FTIR graphs of aerogel particles, fabric electrosprayed 


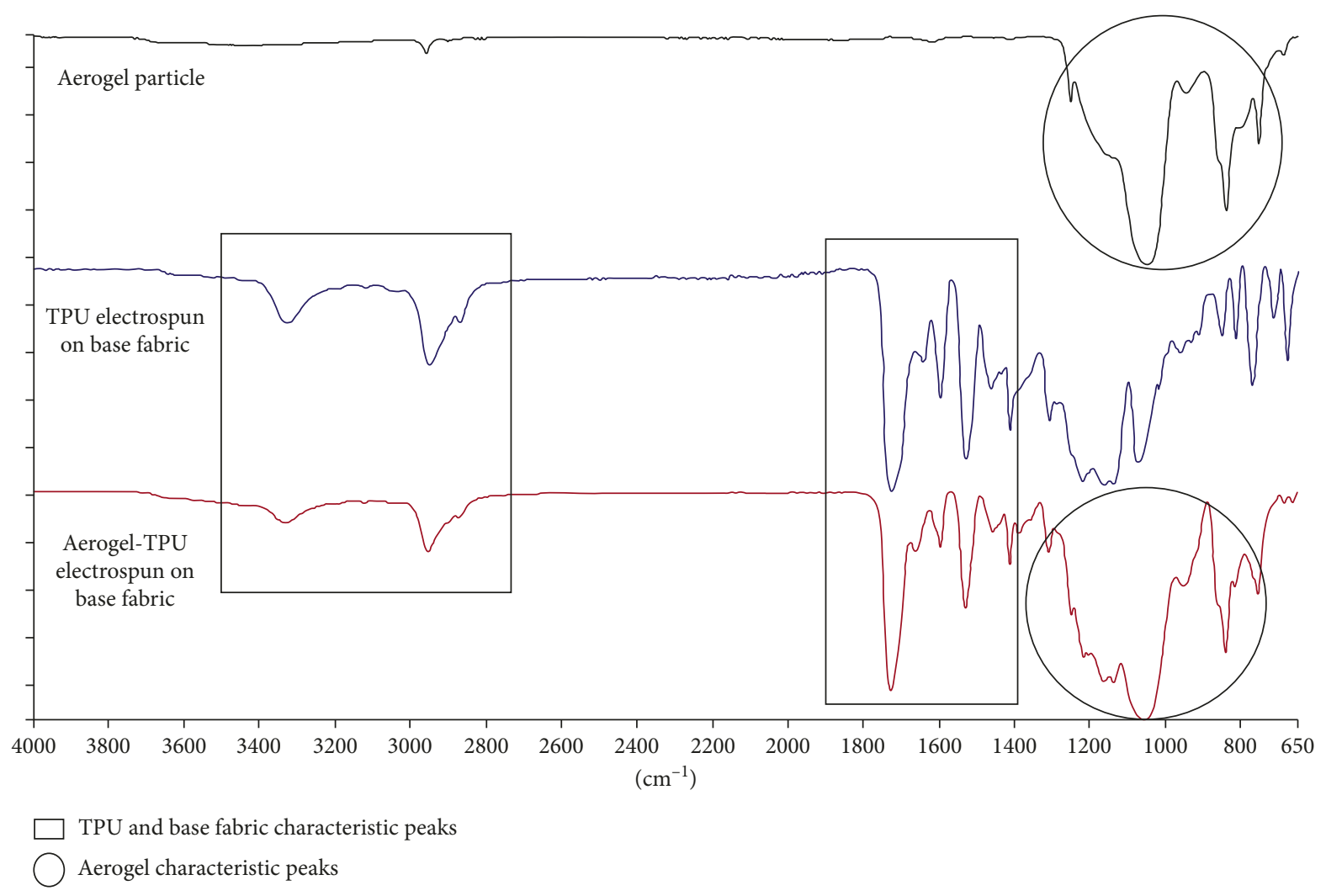

FIGURE 5: FTIR spectra of aerogel particle, polymer spun, and polymer-aerogel-mixed electrosprayed fabric sample.

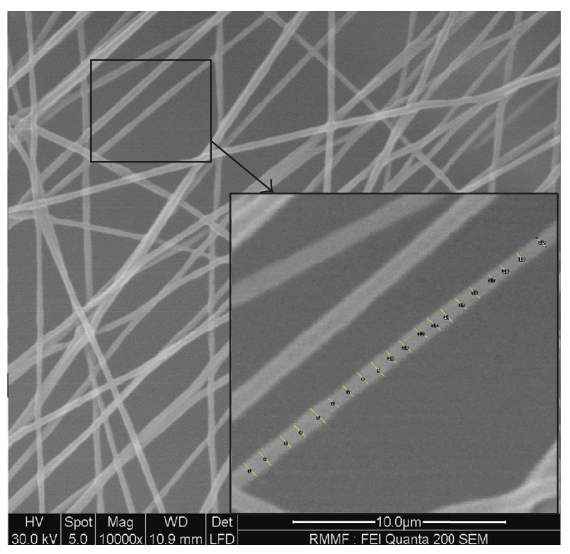

(a)

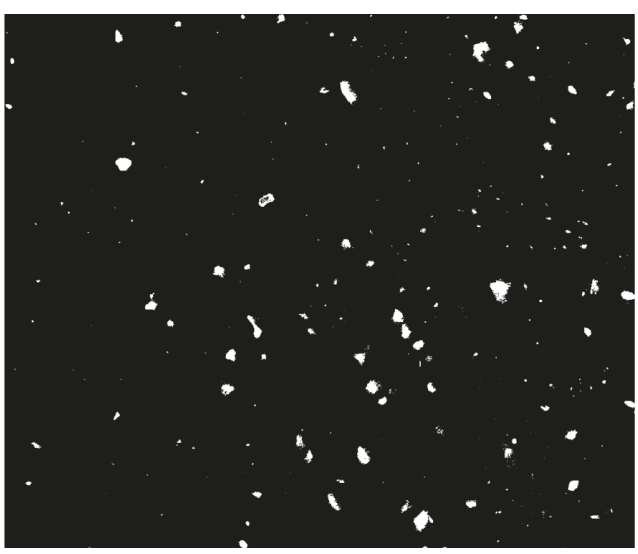

(b)

Figure 6: Measurement of fibre diameter (a) and particle size (b) by using ImageJ.

only with the TPU polymer, and abovementioned electrosprayed sample which contains both aerogel particles and TPU polymer.

From the FTIR spectra, it can be clearly seen that peaks from both aerogel particle and TPU polymer-base fabric are present in the electrosprayed sample. The FTIR spectra of aerogel show an intense peak around $1089 \mathrm{~cm}^{-1}$ which is the typical bending vibration of Si-O [23]. The peak at $843 \mathrm{~cm}^{-1}$ is due to the bending vibration of Si-O $[23,24]$, and the peak at $948 \mathrm{~cm}^{-1}$ is for the stretching vibration of Si-OH [25]. All these peaks can also be seen at the electrospun sample which evidences that aerogel particles were electrosprayed on base fabric by the TPU polymer droplets. The amount of particles transferred by the spraying method was higher than the electrospinning method.

3.2. Fibre Diameter and Particle Size. The SEM images were converted to 8 bit bmp images to analyze the particle size and fibre diameter. The scale bar shown in each SEM image was measured and taken to set scale in ImageJ. Fibre diameter was measured in 20 different positions as shown in Figure 6(a). 


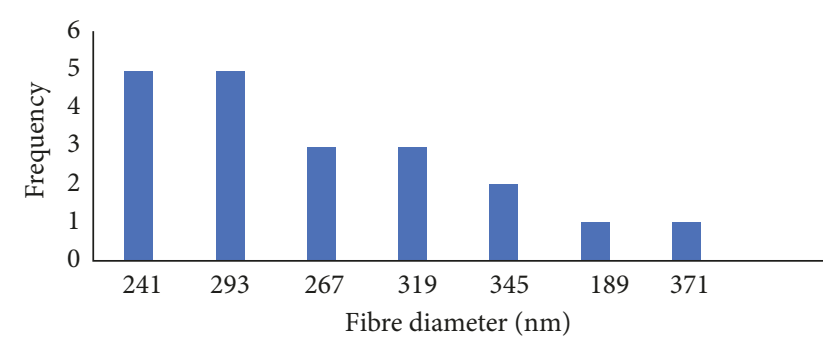

FIGURE 7: Diameter of the produced nanofibre on top of aerogel particles.

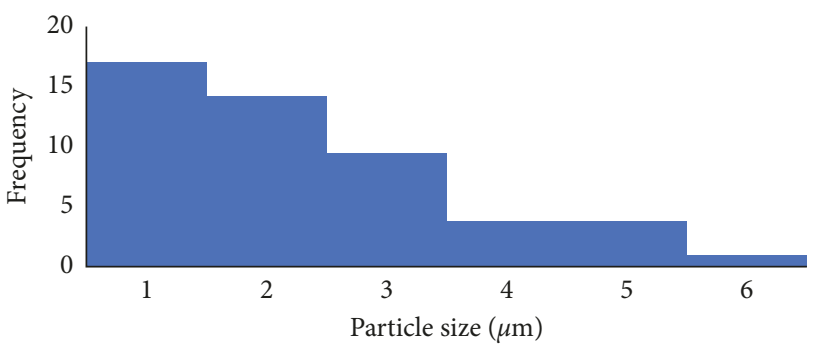

Figure 8: Particle size of transferred aerogel particles.

For particle size measurement, the threshold of the SEM image was first adjusted to nullify the noises other than particles themselves as shown in Figure 6(b). The diameter of the electrospun nanofibre had seen uniform and mostly regular without beads formation. The histogram in Figure 7 shows that the fibre diameter mostly laid between 241 and $293 \mathrm{~nm}$. The diameter ranged from $189 \mathrm{~nm}$ to $368 \mathrm{~nm}$ with an average value of $270 \mathrm{~nm}$.

In the case of the particle size of transferred aerogel particles, it was found that mostly the smaller sized particles were transferred to the base textiles. The average particle size of most of the particles ranges from 1 to $3 \mu \mathrm{m}$ as seen in Figure 8.

\section{Conclusion}

This paper demonstrates an innovative technique to apply particulate material, such as aerogel particles, on textile surface by using the needleless electrospinning/ electrospraying method. A curved wire was used inside the syringe tip as an electrode to charge the particles and polymer mix to generate electric force for discharging of the particles. The SEM micrograph of resultant electrospun web revealed that aerogel particles were transferred to the fabric surface and bonded by the nanofibre web. The electrosprayed surface showed no such fibre deposition, but the higher amount of particle transfer was evident. The findings have numerous opportunities to be utilized for the application of aerogel and other particulate materials on textile or other surfaces using the needleless electrospinning/spraying technique.

\section{Data Availability}

The data used to support the findings of this study are available from the corresponding author upon request.

\section{Conflicts of Interest}

The authors declare that they have no conflicts of interest.

\section{Acknowledgments}

The authors would like to thank Mister Martin Gregory for his support regarding instruments and testings. The authors also thank Dr. Xin Wang for his valuable advice.

\section{Supplementary Materials}

A video file, showing needleless electrospinning of aerogel particle, has been added as supplementary data. (Supplementary Materials)

\section{References}

[1] F. Anton, "Process and apparatus for preparing artificial threads," Google Patents, 1934.

[2] W. Simm, C. Gosling, R. Bonart, and B. V. Falkai, "Fibre fleece of electrostatically spun fibres and methods of making same," Google Patents, 1979.

[3] M. Yu, R. H. Dong, X. Yan et al., "Recent advances in needleless electrospinning of ultrathin fibers: from academia to industrial production," Macromolecular Materials and Engineering, vol. 302, no. 7, pp. 1-19, 2017.

[4] D. Wu, X. Huang, X. Lai, D. Sun, and L. Lin, "High throughput tip-less electrospinning via a circular cylindrical electrode," Journal of Nanoscience and Nanotechnology, vol. 10, no. 7, pp. 4221-4226, 2010.

[5] N. Thoppey, J. Bochinski, L. Clarke, and R. Gorga, "Edge electrospinning for high throughput production of quality nanofibres," Nanotechnology, vol. 22, no. 34, p. 345301, 2011.

[6] S. Tang, Y. Zeng, and X. Wang, "Splashing needleless electrospinning of nanofibres," Polymer Engineering \& Science, vol. 50, no. 11, pp. 2252-2257, 2010.

[7] O. Jirsak, F. Sanetrnik, D. Lukas, V. Kotek, L. Martinova, and J. Chaloupek, "Method of nanofibres production from a polymer solution using electrostatic spinning and a device for carrying out the method," Google Patents, 2009.

[8] B. Lu, Y. Wang, Y. Liu et al., "Superhigh-throughput needleless electrospinning using a rotary cone as spinneret," Small, vol. 6, no. 15, pp. 1612-1616, 2010.

[9] N. M. Thoppey, J. R. Bochinski, L. I. Clarke, and R. E. Gorga, "Unconfined fluid electrospun into high quality nanofibres from a plate edge," Polymer, vol. 51, no. 21, pp. 4928-4936, 2010.

[10] H. U. Shin, Y. Li, A. Paynter, K. Nartetamrongsutt, and G. G. Chase, "Vertical rod method for electrospinning polymer fibers," Polymer, vol. 65, pp. 26-33, 2015.

[11] Y. Liu and J.-H. He, "Bubble electrospinning for mass production of nanofibres," International Journal of Nonlinear Sciences and Numerical Simulation, vol. 8, no. 3, pp. 393-396, 2007.

[12] X. Wang, H. Niu, T. Lin, and X. Wang, "Needleless electrospinning of nanofibres with a conical wire coil," Polymer Engineering \& Science, vol. 49, no. 8, pp. 1582-1586, 2009.

[13] P. Pokorny, E. Kostakova, F. Sanetrnik et al., "Effective AC needleless and collectorless electrospinning for yarn production," Physical Chemistry Chemical Physics, vol. 16, no. 48, pp. 26816-26822, 2014. 
[14] J. Wang, J. Kuhn, and X. Lu, "Monolithic silica aerogel insulation doped with $\mathrm{TiO}_{2}$ powder and ceramic fibers," Journal of Non-Crystalline Solids, vol. 186, pp. 296-300, 1995.

[15] X. Yang, Y. Sun, D. Shi, and J. Liu, "Experimental investigation on mechanical properties of a fiber-reinforced silica aerogel composite," Materials Science and Engineering: A, vol. 528, no. 13-14, pp. 4830-4836, 2011.

[16] A. Shaid, L. Wang, and R. Padhye, "The thermal protection and comfort properties of aerogel and PCM-coated fabric for firefighter garment," Journal of Industrial Textiles, vol. 45, no. 4, pp. 611-625, 2015.

[17] A. Shaid, M. Furgusson, and L. Wang, "Thermophysiological comfort analysis of aerogel nanoparticle incorporated fabric for fire fighter's protective clothing," Chemical and Materials Engineering, vol. 2, no. 2, pp. 37-43, 2014.

[18] L. Jin, K. Honga, and K. Yoona, "Effect of aerogel on thermal protective performance of firefighter clothing," Journal of Fiber Bioengineering and Informatics, vol. 6, no. 3, pp. 315-324, 2013.

[19] M. Venkataraman, R. Mishra, D. Jasikova, T. M. Kotresh, and J. Militky, "Thermodynamics of aerogel-treated nonwoven fabrics at subzero temperatures," Journal of Industrial Textiles, vol. 45, no. 3, pp. 387-404, 2014.

[20] L. Li, B. Yalcin, B. N. Nguyen, M. A. B. Meador, and M. Cakmak, "Flexible nanofibre-reinforced aerogel (xerogel) synthesis, manufacture, and characterization," ACS Applied Materials \& Interfaces, vol. 1, no. 11, pp. 2491-2501, 2009.

[21] H. Wu, Y. Chen, Q. Chen, Y. Ding, X. Zhou, and H. Gao, "Synthesis of flexible aerogel composites reinforced with electrospun nanofibres and microparticles for thermal insulation," Journal of Nanomaterials, vol. 10, pp. 1-8, 2013.

[22] Z. Mazrouei-Sebdani, A. Khoddami, H. Hadadzadeh, and M. Zarrebini, "Synthesis and performance evaluation of the aerogel-filled PET nanofibre assemblies prepared by electrospinning," RSC Advances, vol. 5, no. 17, pp. 12830-12842, 2015.

[23] X. Zhou, H. Xiao, J. Feng, C. Zhang, and Y. Jiang, "Preparation and thermal properties of paraffin/porous silica ceramic composite," Composites Science and Technology, vol. 69, no. 7, pp. 1246-1249, 2009.

[24] Z. Xiangfa, X. Hanning, F. Jian, Z. Changrui, and J. Yonggang, "Preparation, properties and thermal control applications of silica aerogel infiltrated with solid-liquid phase change materials," Journal of Experimental Nanoscience, vol. 7, no. 1, pp. 17-26, 2012.

[25] B. Shokri, M. A. Firouzjah, and S. Hosseini, "FTIR analysis of silicon dioxide thin film deposited by metal organic-based PECVD," in Proceedings of the 19th international symposium on plasma chemistry society, Bochum, Germany, July 2009. 


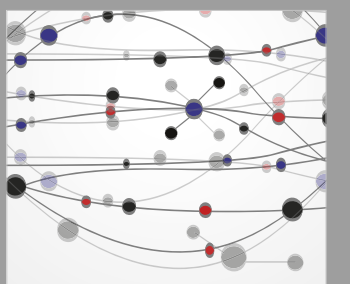

The Scientific World Journal
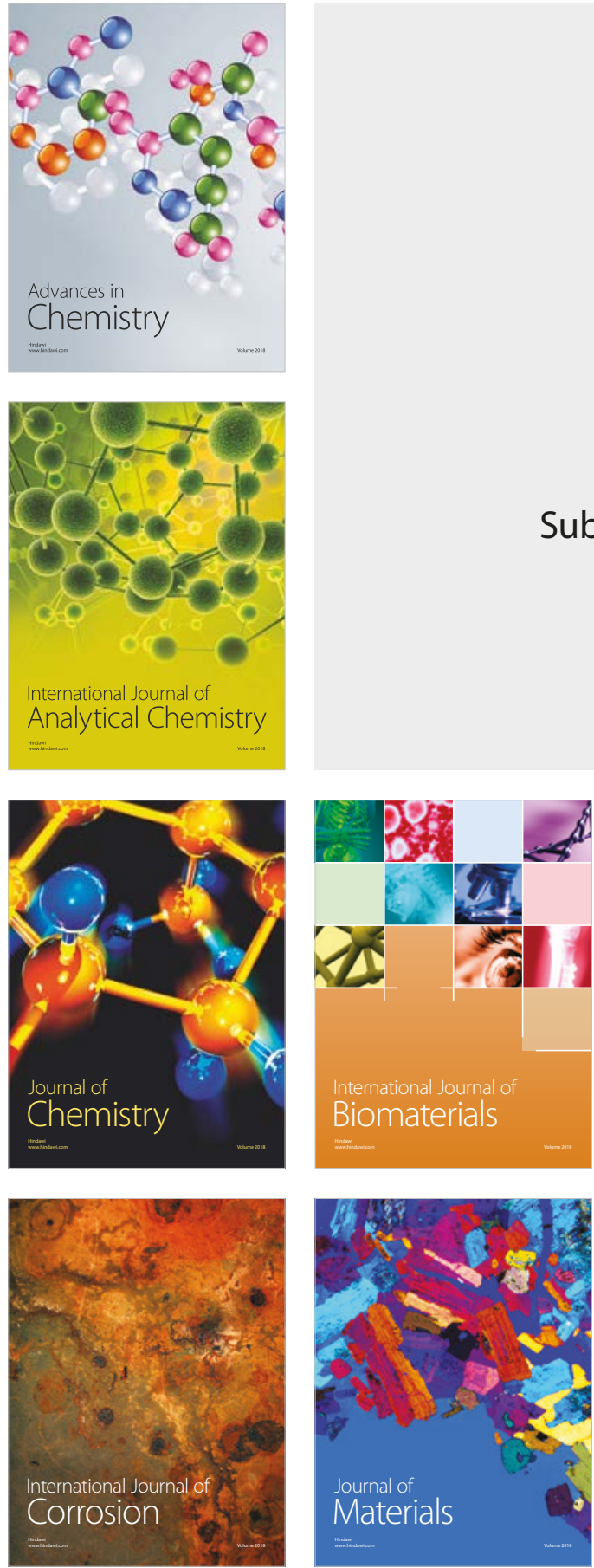

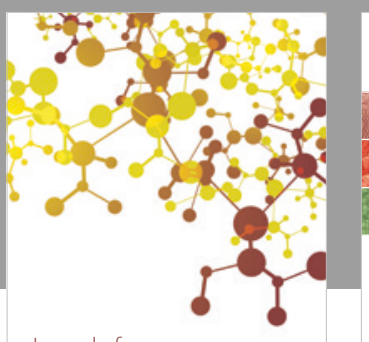

Journal of

Applied Chemistry
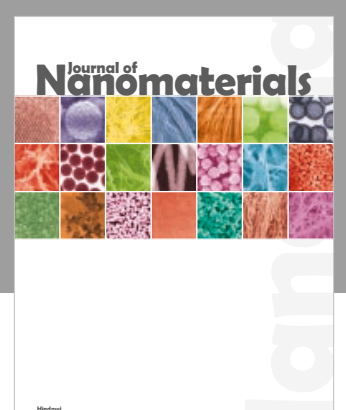

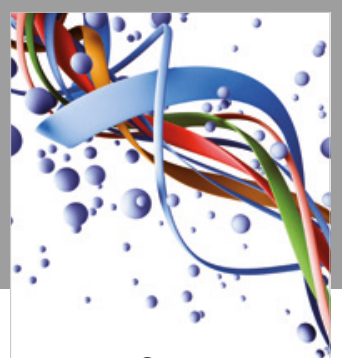

Scientifica

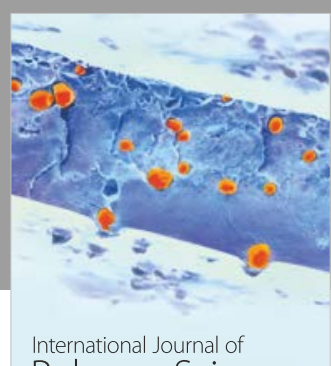

Polymer Science

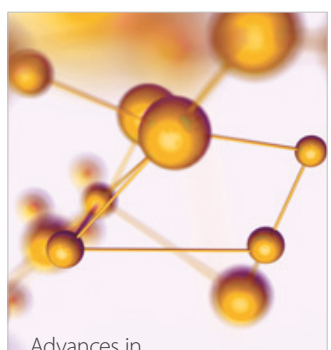

Physical Chemistry
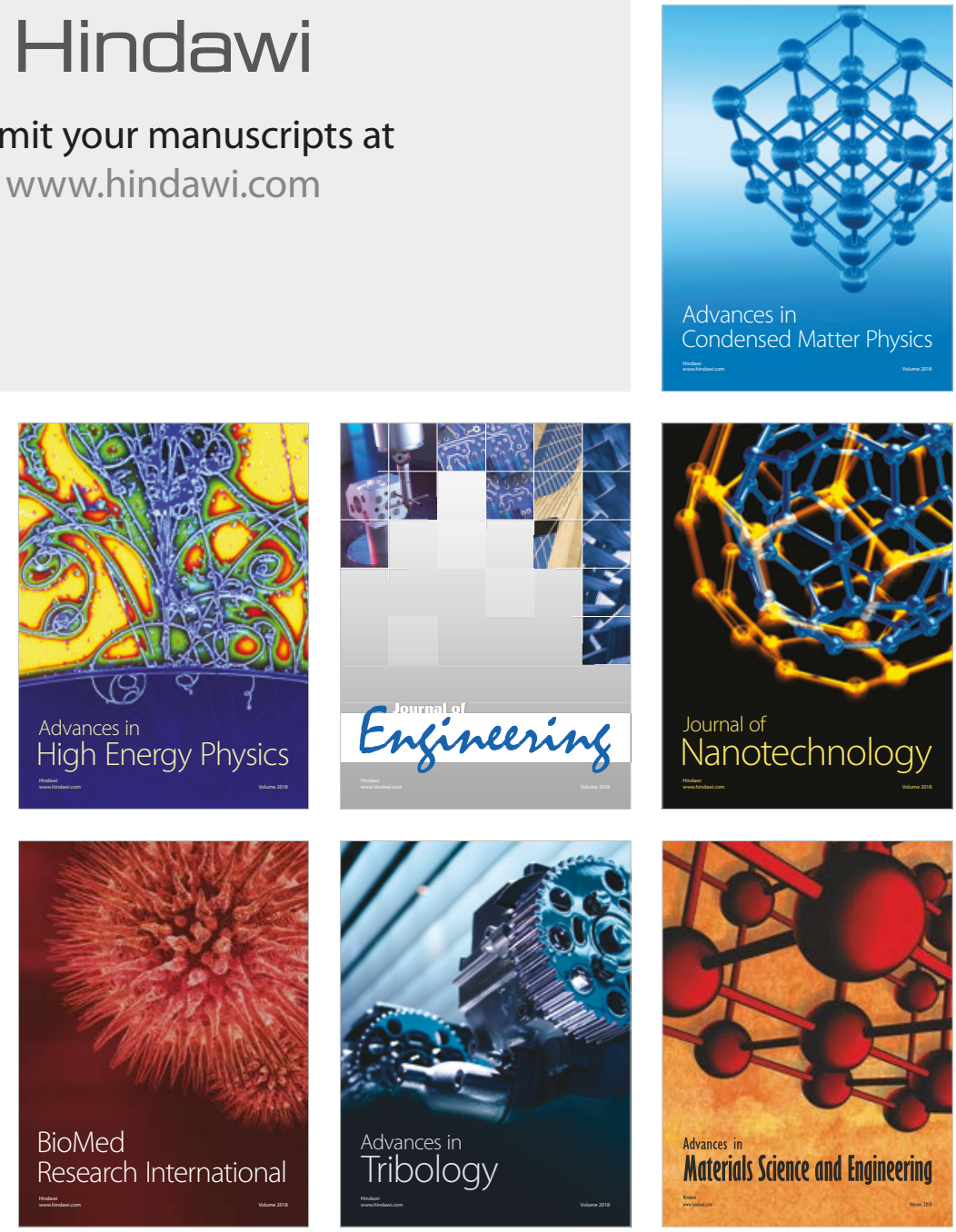